\title{
ARTICLE \\ The gut microbiota mediates reward and sensory responses associated with regimen-selective morphine dependence
}

\author{
Kevin Lee ${ }^{1}$, Helen E. Vuong ${ }^{2}$, David J. Nusbaum (D) ${ }^{2}$, Elaine Y. Hsiao ${ }^{2}$, Christopher J. Evans ${ }^{1,3}$ and Anna M. W. Taylor (iD ${ }^{4}$
}

\begin{abstract}
Opioid use for long-term pain management is limited by adverse side effects, such as hyperalgesia and negative affect. Neuroinflammation in the brain and spinal cord is a contributing factor to the development of symptoms associated with chronic opioid use. Recent studies have described a link between neuroinflammation and behavior that is mediated by a gut-brain signaling axis, where alterations in indigenous gut bacteria contribute to several inflammation-related psychopathologies. As opioid receptors are highly expressed within the digestive tract and opioids influence gut motility, we hypothesized that systemic opioid treatment will impact the composition of the gut microbiota. Here, we explored how opioid treatments, and cessation, impacts the mouse gut microbiome and whether opioid-induced changes in the gut microbiota influences inflammation-driven hyperalgesia and impaired reward behavior. Male C57B/6/J mice were treated with either intermittent or sustained morphine. Using 16S rDNA sequencing, we describe changes in gut microbiota composition following different morphine regimens. Manipulation of the gut microbiome was used to assess the causal relationship between the gut microbiome and opioid-dependent behaviors. Intermittent, but not sustained, morphine treatment was associated with microglial activation, hyperalgesia, and impaired reward response. Depletion of the gut microbiota via antibiotic treatment surprisingly recapitulated neuroinflammation and sequelae, including reduced opioid analgesic potency and impaired cocaine reward following intermittent morphine treatment. Colonization of antibiotic-treated mice with a control microbiota restored microglial activation state and behaviors. Our findings suggest that differing opioid regimens uniquely influence the gut microbiome that is causally related to behaviors associated with opioid dependence.
\end{abstract}

Neuropsychopharmacology (2018) 43:2606-2614; https://doi.org/10.1038/s41386-018-0211-9

\section{INTRODUCTION}

Opioids are the strongest and most effective analgesics available for acute pain management, but long-term usage is limited by adverse side effects such as hyperalgesia, and addiction. Strategies that target the negative effects of opioids while retaining their analgesic properties would enhance the drug's effectiveness in treating chronic pain and mitigate unwanted physiological consequences of chronic opioid use [1, 2]. Prolonged opioid exposure is associated with the gradual loss of analgesic potency (tolerance) and a paradoxical increase in pain sensitivity during drug withdrawal (hyperalgesia). Tolerance can also develop to the rewarding effects of opioid drugs, and is driven by opponent processes that impair reward processing resulting in negative affect during prolonged periods of drug abstinence [2]. A growing body of research points to neuroinflammation as a contributor to centrally mediated adverse effects of opioids, representing a potential target for symptom relief. Systemic morphine administration activates the glial-driven inflammatory cascade within the dorsal horn of the spinal cord [3, 4]. Genetic or pharmacological abrogation of microglial activation-either systemically or locally within the spinal cord-prevents opioid-induced hyperalgesia without affecting tolerance $[5,6]$. Opioid-induced microglial activation also extends rostrally throughout the brain, including the hippocampus, thalamus, striatum, and midbrain [7, 8]. Recently, we have shown opioid-induced activation of microglia in the ventral tegmental area (VTA) leads to a reduction in dopaminedependent reward behavior via a microglia-brain-derived neurotrophic factor (BDNF) signaling pathway [9]. Inhibition of VTA dopamine neuron activity elicits depression-like phenotypes in mice, and deficient midbrain dopamine signaling is a hallmark of anhedonia [10, 11]. Thus, we posit that the glial-driven deficit in dopamine signaling is a key contributor to the impaired reward behavior of opioid withdrawal [1]. In fact, inflammation-driven hypodopaminergia is a characteristic of many conditions associated with impaired reward behavior [12], and may represent a pivotal phenomenon driving the anhedonic states of both drug withdrawal and clinical depression.

Symbiotic bacteria from the indigenous gut microbiota contribute to the development and function of microglia in the brain, and perturbations in the gut microbiota are associated with abnormal microglial reactivity $[13,14]$. Changes in the gut microbiota are also associated with alterations in host behaviors and psychological disorders, including depression and anxiety [15]. Fecal samples isolated from depressed patients showed significant alterations in gut flora compared to healthy controls [16]. Recolonizing the gut of healthy mice with fecal samples

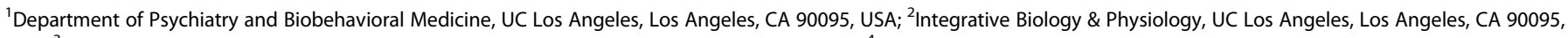

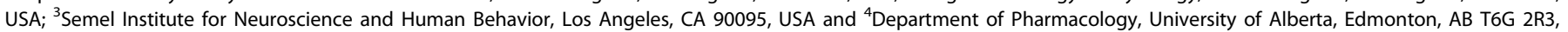
Canada

Correspondence: Anna M. W. Taylor (ataylor1@ualberta.ca)

Received: 19 February 2018 Revised: 17 August 2018 Accepted: 20 August 2018

Published online: 10 September 2018 
isolated from depressed human patients induced depression-like behaviors in mice (ibid). Thus, the gut microbiome may serve as an important mediator of inflammation and negative affect.

Systemic opioids impact gut motility markedly by either decreasing gut motility during drug onset, or increasing gut motility during withdrawal $[17,18]$. Changes in gut motility can influence the composition of the gut microbiota [19]. Given these two findings, it is reasonable to expect systemic opioids influence the gut microbiota and that alterations will be drug and drug-regimen specific. Emerging evidence in both animal and human studies suggests opioids can markedly modify the gut microbiota [20-22].

The goal of this current study is twofold. First, given that opioids can influence gut motility either directly or indirectly, it remains to be seen how drug use and drug cessation (withdrawal) impacts the gut microbiota. Using $16 \mathrm{~S}$ rDNA sequencing of bacterial communities within fecal samples, we describe changes in the gut microbiota following opioid exposure and following withdrawal. Second, we explore the causative connection between opioid-induced changes in the gut microbiota with inflammation and maladaptive behaviors associated with dependence and withdrawal.

\section{MATERIALS AND METHODS}

Animals

Male C57BI/6J mice (The Jackson Laboratory, Bar Harbor, ME) 6-8weeks old at the beginning of experimentation were used. Animals were housed in groups of two to four in autoclaved, ventilated, microisolator caging, and kept on a 12-h light/dark cycle with food and water available ad libitum. All behavioral tests were performed during the light phase, and equipment was disinfected prior to use. All procedures were conducted in accordance with the National Institutes of Health Guide for the Care of Use of Laboratory Animals and were approved by the UCLA Institutional Animal Care and Use Committee.

\section{Drug treatment}

Mice were made opioid-dependent by two paradigms: (1) intermittent morphine consisting of twice-daily injections of escalating doses of morphine sulfate $(10,20,30,40 \mathrm{mg} / \mathrm{kg}$, intraperitoneal (i.p.); National Institutes on Drug Abuse (NIDA) Drug Supply, Bethesda, MD) for 4 days, or (2) continuous morphine consisting of a surgically implanted subcutaneous morphine sulfate pellet ( $25 \mathrm{mg}$; NIDA Drug Supply). Control animals (drugnaive) received twice-daily vehicle (saline, $0.9 \% \mathrm{NaCl}$, i.p.) injections or a subcutaneous vehicle pellet (NIDA Drug Supply) in lieu of the drug. Antibiotic-treated animals were provided ampicillin $(1 \mathrm{~g} / \mathrm{L}$; Sigma Aldrich, St.Louis, MO), vancomycin ( $0.5 \mathrm{~g} / \mathrm{L}$; Chem-Impex, Wood Dale, IL), and neomycin (100 mg/L; Chem-Impex) in sterile drinking water for at least 4 days. This cocktail of antibiotics was selected to significantly deplete the microbiome [23], and does not mirror clinical antibiotic doses.

\section{S rDNA sequencing}

Fresh fecal samples were collected from awake animals housed in separate cages, and flash frozen on dry ice. Samples were stored at $-80^{\circ} \mathrm{C}$ until DNA extraction. The libraries were generated according to methods adapted from [24]. Bacterial genomic DNA was extracted using the Powersoil DNA Isolation Kit (Mo Bio Laboratories, Carlsbad, CA), and the V4 region of the 16S rRNA gene was PCR amplified in triplicate using individually barcoded universal primers and purified using Qiaquick PCR purification kit (Qiagen). The purified PCR product was pooled in equal molar concentrations and sequenced by Laragen, Inc. using the Illumina MiSeq platform and $2 \times 250 \mathrm{bp}$ reagent kit for paired-end sequencing. Operational taxonomic units (OTUs) were selected using open reference OTU picking based on $97 \%$ sequence similarity to the Greengenes 13_5 database. Taxonomy assignment and rarefaction were performed using QIIME 1.9.1 using 36,000 reads per sample.

Immunohistochemistry

Following fecal sampling, animals were deeply anesthetized with isoflurane. Whole brain and spinal cord sections between L4 and L6 vertebrae were isolated from the animal and immediately immersed in $4 \%$ paraformaldehyde (PFA) in $0.1 \mathrm{M}$ phosphate buffer (PB) for $48 \mathrm{~h}$ at $4{ }^{\circ} \mathrm{C}$. This was followed by immersion in a $30 \%$ sucrose solution (in $0.1 \mathrm{M} \mathrm{PB}$ ) for another $48 \mathrm{~h}$ at $4{ }^{\circ} \mathrm{C}$. Tissue was then frozen in OCT embedding compound (Tissue-Plus OCT Compound, Fisher Scientific, Waltham, MA) and sectioned at $50 \mu \mathrm{M}$ on a cryostat (Leica CM3050 S, Leica Biosystems, Buffalo Grove, IL), collected in $0.1 \mathrm{M}$ phosphate buffered saline (PBS). Free-floating sections were stained with anti-IBA1 (1:2000; Wako Chemicals USA, Richmond, VA), and subsequently stained with goat anti-rabbit Alexa 488 (1:1000; Molecular Probes, Fisher Scientific, Waltham, MA). Sections were mounted on glass slides and sealed with Vectashield (Vector Laboratories, Burlingame, CA).

Images were acquired on an Olympus BX51 fluorescent scope (Olympus; Tokyo, Japan), using a 20x objective connected to a CCD camera. ImageJ software (National Institutes of Health, Bethesda, MD) was used to quantify the number of microglia and cell body size. For cell counts, only clearly labeled and in-focus cells were counted in the entire optical region. During activation, microglia undergo significant morphological changes transitioning from highly ramified structures with small cell bodies to larger ameboid cells with fewer branches [25]. Here, we use changes in cell body size to assess changes in the activation state of microglial cells. Cell body size was measured by determining the distance between the two most distant points of the cell body. Approximately 12 cells per section were measured. For both the cell counts and cell body size measurement, 3-7 sections per animal were collected and averaged.

Conditioned place preference (CPP)

CPP was conducted using an unbiased, counter balanced, twochamber apparatus. Conditioning sessions consisted of mice receiving four trials of cocaine hydrochloride $(10 \mathrm{mg} / \mathrm{kg}$, i.p. NIDA Drug Supply) and four trials of saline vehicle (i.p.) on alternating days. Animals were confined to the chambers for 15 min following injection, and immediately returned to their home cages. On the post-conditioning day (day 9), animals were allowed free access to both chambers in a drug free state, and the time spent in the drug-paired chamber was measured over $15 \mathrm{~min}$.

Tail withdrawal assay

Mice were habituated to the testing room and restraint apparatus prior to the experiment. On the test day, mice were gently restrained, and the distal $5 \mathrm{~cm}$ of the tail was submerged in $49^{\circ} \mathrm{C}$ water until a vigorous withdrawal reaction was observed. The time between tail submersion and withdrawal response was recorded. A cut-off of $15 \mathrm{~s}$ was imposed to avoid tissue damage. Three baseline measurements were recorded. Immediately following the last baseline measurement, mice received escalating doses of morphine $(1,3,10,30,100 \mathrm{mg} / \mathrm{kg}$, i.p.) every $30 \mathrm{~min}$. Tail flick time was measured $10 \mathrm{~min}$ after each injection. The $\mathrm{EC}_{50}$ (concentration of the drug that gives half-maximal response) was extrapolated from the dose-response curves.

Intestinal permeability assay

Mice were treated with intermittent or sustained morphine (or vehicle), as described above. Immediately following the last drug or saline injection (day 4), mice were returned to their home cages without food or water. $12 \mathrm{~h}$ later, mice were orally gavaged with FITC-dextran (Sigma; $100 \mathrm{mg} / \mathrm{mL}$ in PBS) at a dose of $44 \mathrm{mg} / 100 \mathrm{~g}$ body weight. After $4 \mathrm{~h}$, mice were anesthetized with isoflurane 
and $300-400 \mu \mathrm{L}$ of blood collected via cardiac puncture. Serum was isolated and plated on a 96-well plate and read by spectrophotofluorometry with an excitation of $495 \mathrm{~nm}$. FITCdextran concentration was calculated with a standard serially diluted FITC-dextran. Serum from mice not administered with FITC-dextran was used to determine background.

\section{Data analysis}

In all experiments, data were tested for normality, and parametric or non-parametric statistics were used accordingly. Group means were compared using unpaired Student's t-test or Mann-Whitney test. For comparisons using data sets with more than two groups or conditions, a one-way or two-way ANOVA or a Kruskal-Wallis test followed by appropriate post hoc test was used (Graph Pad Prism, La Jolla, CA). Taxonomic comparisons from 16S rDNA sequencing analysis were analyzed by Kruskal-Wallis test with Bonferroni post hoc test. In all experiments, significance threshold was set at $p<0.05$.

\section{RESULTS}

Intermittent, but not sustained, morphine alters microglial morphology, and hyperalgesia

Previous work from our lab and others have found that intermittent morphine treatment activates microglia in both spinal cord and reward-related structures leading to hyperalgesia and impaired dopamine-dependent reward behavior via a BDNFmediated signaling mechanism [5, 9]. Here, we compare the ability of two morphine treatment regimens (intermittent versus sustained) to activate microglia and subsequent changes in opioid response (Fig. 1a).

Both intermittent and sustained morphine treatment regimens resulted in morphine tolerance, as indicated by a rightward shift in the dose-response curve (Fig. $1 \mathrm{~b}, \mathrm{~F}_{\text {treatment }}(1,14)=24.95$, $p=0.0002$ ). There was an impact of surgery on the morphine dose-response curve with the saline pellet group showing a rightward shift in the morphine dose-response curve when compared to the drug and surgery naive group $\left(F_{\text {treatment }}(1,13)=\right.$ $32.33, p=0.04)$. All comparisons are done to the appropriate control (saline injection to morphine injection and saline pellet to morphine pellet) in order to control for this effect. The change in $\mathrm{EC}_{50}$ was greater in the morphine pellet group compared to the morphine injection group when controlling for the effect of surgery $(U=8, p=0.02)$. Intermittent morphine treatment resulted in a significant reduction in baseline withdrawal latencies, indicative of hyperalgesia (Fig. $1 c, t=4.47, \mathrm{df}=14, p=0.0005$ ). However, the baseline withdrawal threshold of sustained morphine-treated mice at the equivalent time-period revealed a significant elevation in thresholds (i.e., analgesia), suggesting that residual morphine analgesia from the subcutaneous pellet remains $(F(3,26)=30.2, p<0.0001)$. Only when the subcutaneous morphine pellet was removed $12 \mathrm{~h}$ prior were baseline withdrawal thresholds reduced below control (saline pellet) levels $(p=0.006)$.

Prolonged opioid treatment also impairs reward processing, a process thought to underlie the anhedonic state associated with drug withdrawal [26]. Here, we compared the ability of intermittent and sustained morphine treatment to impact cocaine-conditioned place preference. As expected, opioid-naive controls (sham pellet and saline injection) displayed robust place preference for the cocaine-paired chamber (Fig. 1d, $F(3,20)_{\text {inter- }}$ mittent $=4.81, p=0.003, F(3,28)$ sustained $=7.5, p=0.0009)$. Pretreatment with intermittent morphine impaired the development of cocaineconditioned place preference $(p=0.83)$, as previously reported for morphine and other opioids $[9,27]$. In contrast, sustained morphine treatment did not impact cocaine-conditioned place preference; mice pretreated with sustained morphine showed significant preference for the cocaine-paired chamber $(p=0.03)$
Changes in microglial morphology in the spinal cord and VTA mirrored the behavioral results discussed above, whereby intermittent morphine significantly altered microglial morphology. Intermittent morphine resulted in significantly larger microglial cell body size in the VTA and dorsal spinal cord compared to both drug-naive controls and sustained morphine treatment group (Fig. 1e, $F(3,176)=43.31, p<0.0001$ ). Sustained morphine treatment did not alter microglial cell body size when compared to saline pellet controls $(p=0.67)$, but removal of the pellet was sufficient to initiate an inflammatory response within $12 \mathrm{~h}$ $(p=0.01)$. No difference in cell number was observed between groups $(\mathrm{F}(3,26)=0.23, p=0.35)$.

Dysbiosis of the gut microbiota in opioid treated mice Bacterial DNA isolated from fecal pellets collected from control and morphine-treated mice were prepared for $16 \mathrm{~S}$ rDNA sequencing (Fig. 2a). Morphine treatment (either intermittent or sustained) did not impact bacterial alpha-diversity, but principal coordinates analysis ( $\mathrm{PCOA}$ ) revealed that microbiota from mice exposed to either intermittent or sustained morphine were discriminable from controls in bacterial beta-diversity (Fig. 2b, c). We identified several bacterial genera in the feces that were altered in animals treated with morphine compared to drug-naive controls (Fig. 2d-f). Mice treated with intermittent morphine had increased relative abundance of Ruminococcus spp. $(H=14.57, p$ $=0.003)$ and decreased Lactobacillus spp. $(H=12.21 p=0.02)$ compared to saline injection controls. Mice treated with sustained morphine did not show significant changes in Ruminococccus spp. ( $p=0.17)$ or Lactobacillus spp. $(p=0.39)$ compared to saline pellet controls, but had increased relative abundances in genera such as Clostridium spp. $(\mathrm{H}=7.74, p=0.01)$ and the family Rikenellaceae $(\mathrm{H}=7.23, p=0.0004)$. Finally, intermittent morphine, but not sustained morphine treatment, resulted in significant increase gut permeability (Fig. S1). Overall, these results reveal that both intermittent and sustained morphine treatment rendered statistically significant changes in gut bacterial profiles, but that the morphine treatment regimen has differential effects on the composition of the gut microbiota.

Manipulation of the gut microbiome influences neuroinflammation and opioid-dependent behaviors

We next tested the effect of depleting the gut microbiome on microglial activation and opioid-related behaviors (Fig. 3a). Fourday treatment with the antibiotic cocktail resulted in significant depletion of the gut microbiota, as indicated by a significant reduction in bacterial alpha-diversity (Fig. 3b, $\mathrm{t}=6.25$, $\mathrm{df}=20, p<$ 0.0001 ). Antibiotic-treated opioid-naive mice exhibited an unanticipated rightward shift in the morphine dose-response curve and doubling of the $\mathrm{EC}_{50}$ dose when compared to water-treated controls. Both treatment with antibiotics alone or in combination with intermittent morphine produced a rightward shift in the dose-response curve compared to controls (Fig. 3c, $F_{\text {treatment }}(2,15)$ $=17.29, p \leq 0.0001)$. Antibiotic mice did not demonstrate hyperalgesia unless they were also treated with morphine (Fig. $3 \mathrm{~d}, \mathrm{H}=$ 33.12, $p<0.0001)$. Similar to intermittent morphine mice, antibiotic-treated mice did not demonstrate significant preference for either the cocaine- or saline-paired chamber (Fig. 3e, F(1.759, $26.39)=4.09, p=0.16)$. Increased microglial cell body size in the VTA and dorsal spinal cord was observed in antibiotic-treated mice $\left(\mathrm{F}_{\text {spinal cord }}(2,13)=27.46, p=<0.0001, \mathrm{~F}_{\mathrm{VTA}}(2,15)=7.74, p=\right.$ $0.006)$. No significant difference in microglial cell body size were observed between antibiotic-treated and morphine-dependent mice. (Fig. 3f, $p_{\text {spinal cord }}=0.95, p_{V T A}=0.45$ ).

Gut recolonization with an "opioid" microbiome Finally, to determine whether the intermittent morphineassociated microbiota is sufficient to produce an opioiddependent phenotype in drug-naive animals, antibiotic-treated 
A

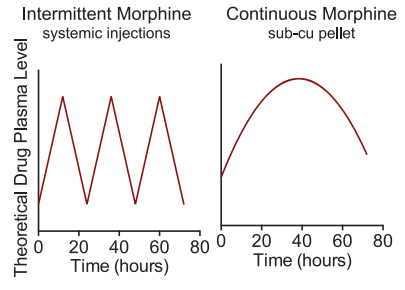

B

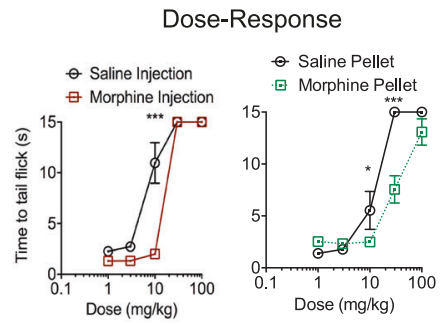

$\mathrm{EC}_{50}$

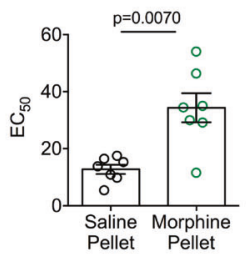

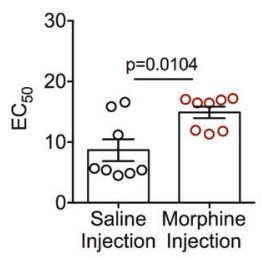

Evoked Pain

Immunostain

C
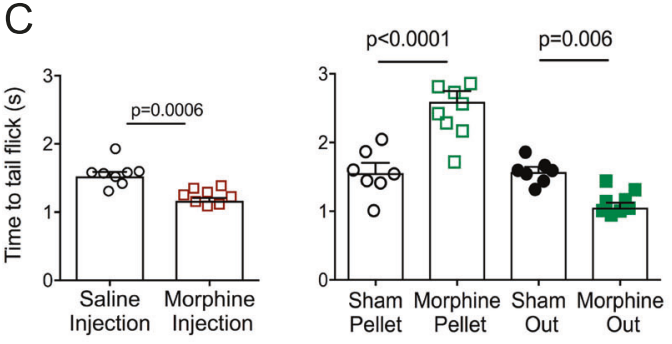

D
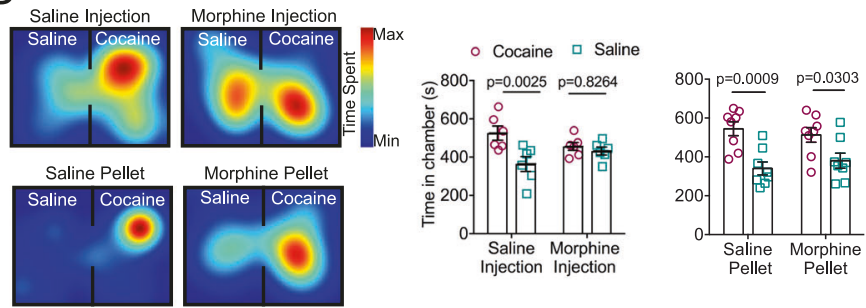

E

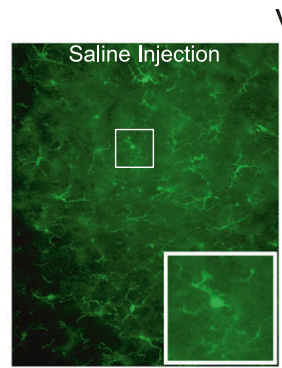

VTA
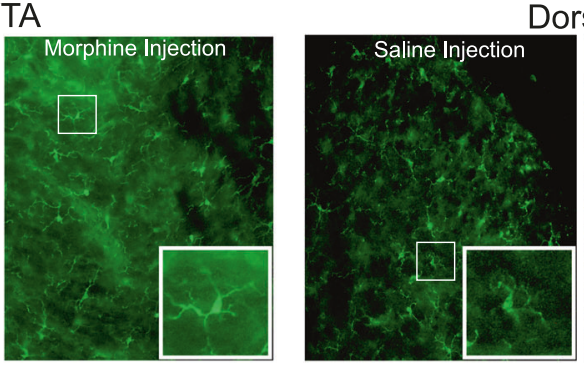

Dorsal Horn
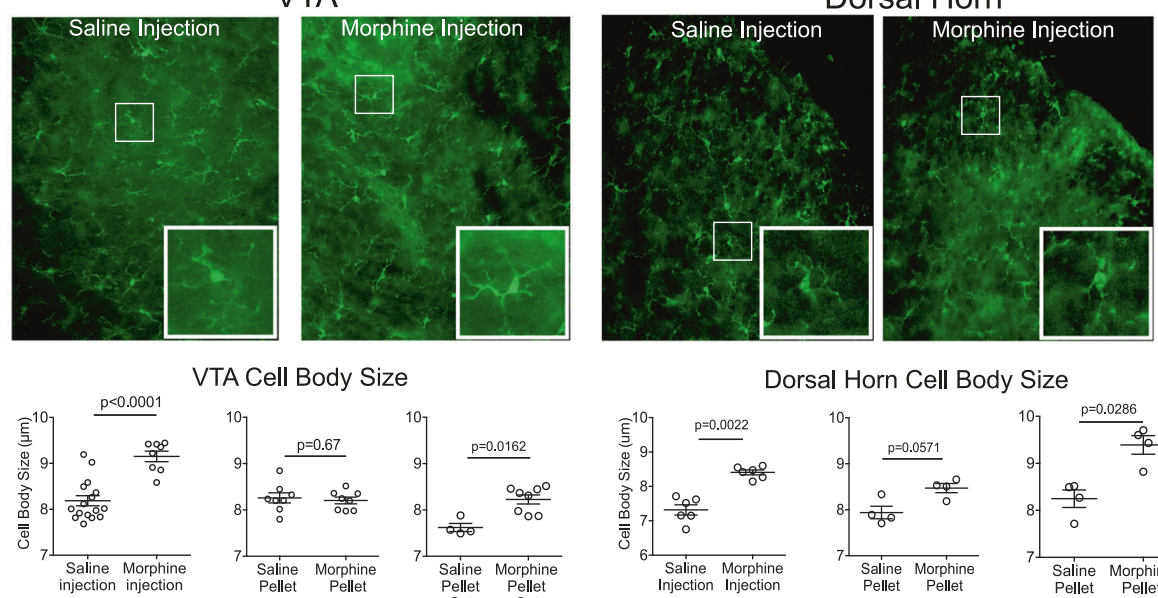

VTA Cell Body Size
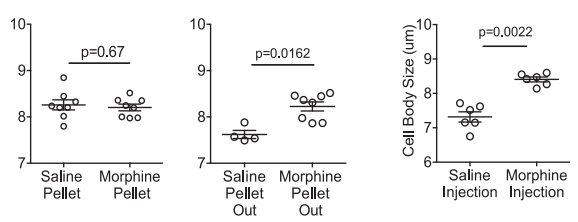

Dorsal Horn Cell Body Size

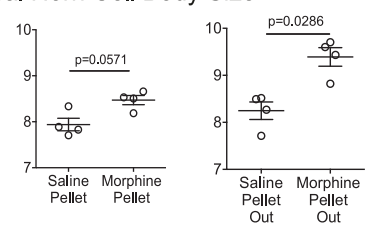

Fig. 1 Morphine withdrawal alters microglial morphology, hyperalgesia, and impaired reward behavior. a Experimental design. Top: theoretical drug plasma levels in intermittent morphine and continuous morphine groups over four days. Bottom: timeline of experiments, starting with four-day opioid treatment regimen followed by (1) ten days of cocaine-conditioned place preference testing, (2) evoked pain testing, or (3) immunostaining. b Morphine dose-response assayed using thermal tail withdrawal compared in animals pretreated with saline injection or pellet and morphine injection or pellet (error bars $=$ S.E.M., ${ }^{*} p=0.04,{ }^{* * *} p<0.0001, n=7-8$ ). Right histograms show $\mathrm{EC}_{50}$ values for treatment groups compared with Mann-Whitney tests (error bars =S.E.M., $n=7-8$ ). c Baseline thermal withdrawal thresholds assayed $12 \mathrm{~h}$ after the last morphine injection in animals pretreated with saline or morphine injection, saline or morphine pellet, or morphine pellet $24 \mathrm{~h}$ after pellet removal. Groups compared with Student's $t$-test or one-way ANOVA (error bars = S.E.M., $n=7-8$ ). $\mathbf{d}$ Conditioned place preference to cocaine in animals pretreated with intermittent and sustained morphine. Left: representative heat maps illustrating time spent in either saline-paired or cocaine-paired chamber. Right: Group means compared using one-way ANOVA followed by Sidak's multiple comparison tests. e Impact of sustained and intermittent morphine on microglia morphology. Top: representative immunomicrographs of microglia from saline injection and morphine injection groups in the ventral tegmental area (VTA) and dorsal spinal cord (inset box $=50 \mu \mathrm{m}$ by $50 \mu \mathrm{m}$ ). Bottom: Graphs represent quantification of 3-4 brain and spinal cord sections per animal. Groups were compared using one-way ANOVA (error bars $=$ S.E.M, $n=4-16$ )

mice were transplanted with saline or morphine microbiota (Fig. 4a). The saline and morphine microbiota recipients showed similar species richness, which was significantly greater than the antibiotic-treated group (Fig. $4 \mathrm{~b}, \mathrm{~F}(2,30)=6.06, p=0.006$ ). Antibiotic-treated mice that were transplanted with saline control microbiota, but not morphine-associated microbiota exhibited increased baseline thermal withdrawal threshold (Fig. 4c, $d$, $t_{\text {saline }}$ $=3.07, \mathrm{df}=25, \quad p<0.0001 ; \mathrm{t}_{\text {morphine }}=0.39, \mathrm{df}=25, \quad p=0.27$ ) and restored cocaine place preference behavior $(F(3,26)=39.74$, $p=<0.0001)$. Finally, mice with morphine-associated microbiota had increased microglial cell body size in both the midbrain and dorsal horn of the spinal cord compared to naive controls (no intervention) and saline microbiota transplant (Fig. $4 \mathrm{e}$, $\left.\mathrm{H}_{\mathrm{VTA}}=12.63, p=0.0002 ; \mathrm{H}_{\text {spinal cord }}=10.66, p=0.0006\right)$.

\section{DISCUSSION}

While opioids are strong analgesics, their use is hampered by engagement of opponent processes resulting in tolerance, hyperalgesia, and negative affect [28]. We and others have shown 
A

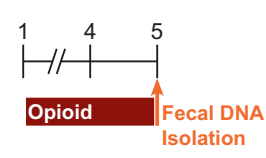

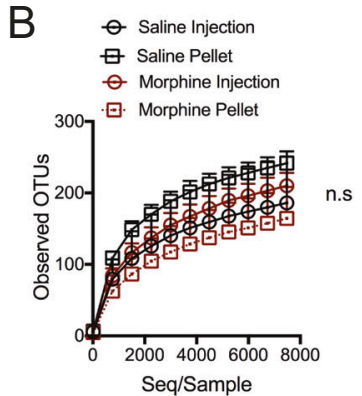

$\mathrm{D}$
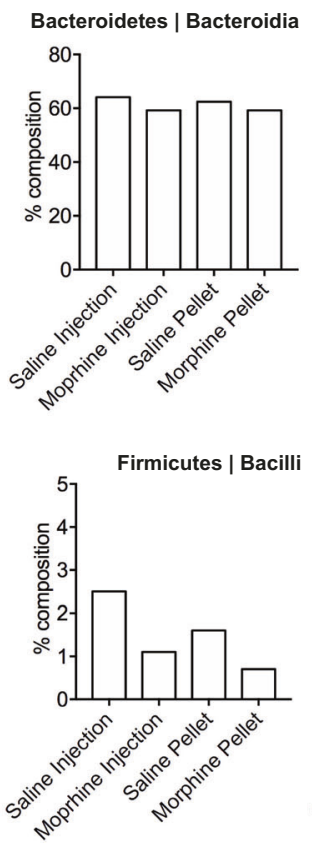

Firmicutes | Clostridia
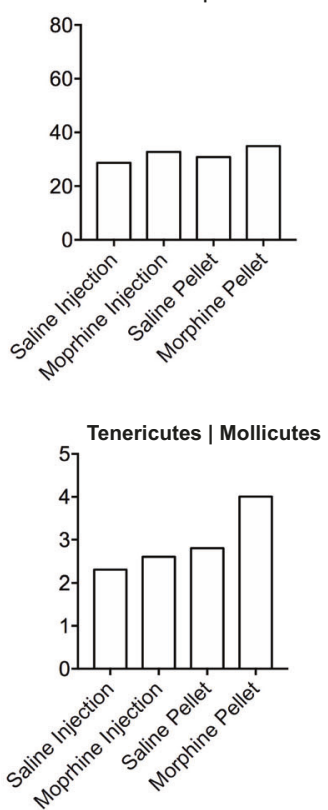

C

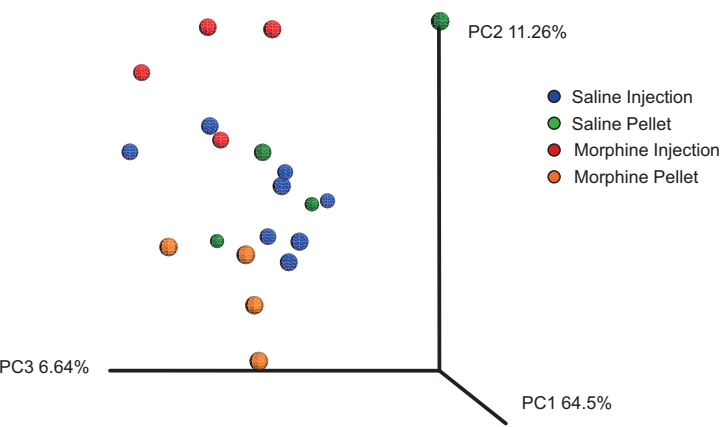

E
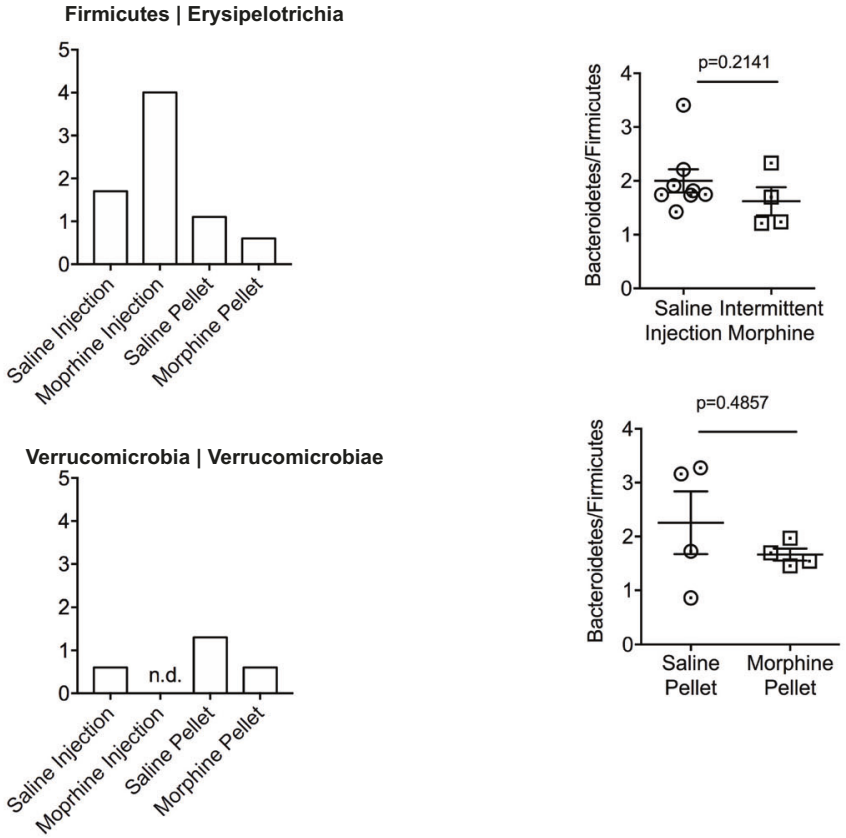

F

Morphine Injection

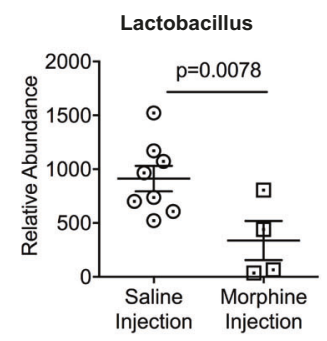

Ruminococcus

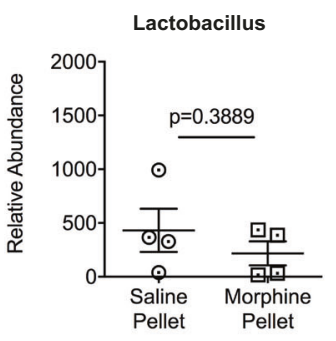

\section{Morphine Pellet}
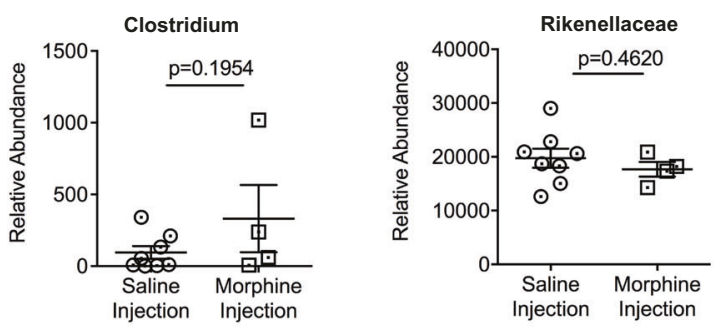
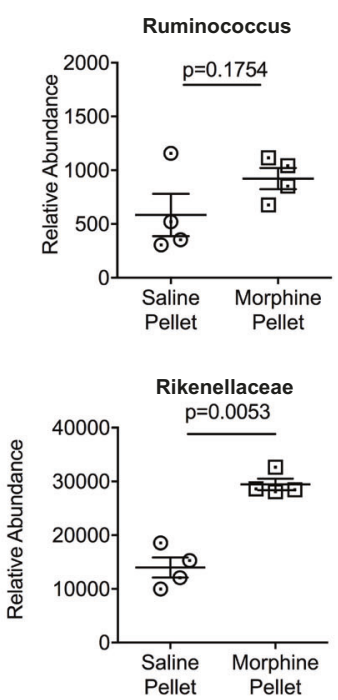

Fig. 2 Intermittent and sustained morphine uniquely influence beta-diversity of the gut microbiota. a Timeline outlining experiment, starting with four-day opioid treatment regimen followed by fecal pellet collection. $\mathbf{b}$ Richness of the gut microbiota is illustrated by rarefaction curves plotting observed taxonomic units (OTUs) versus the number of sequences per treatment group, $n=4-8$ per group. c Principal coordinates analysis of the fecal microbiota from intermittent and sustained morphine-treated mice, $n=4-8$. $\mathbf{d}$ Percent relative abundance of OTUs classified at the class level per treatment group. e Relative abundance (ratio) of the most abundant phyla Bacteroidetes and Firmicutes from samples treated with intermittent or sustained morphine. Groups were compared using a Mann-Whitney test (error bars=S.E.M.). f Relative abundance (number of reads/total reads) of key OTUs in the Lactobacillus, Ruminococcus, Clostridium genera, and the Rikenellaceae family from samples obtained from animals treated with intermittent or sustained morphine. Groups were compared using Kruskal-Wallis followed by Bonferroni test (error bars = S.E.M.) 


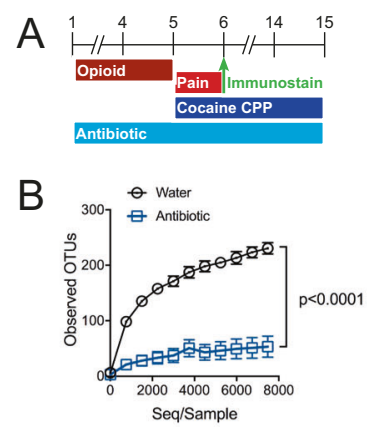

$\mathrm{E}$

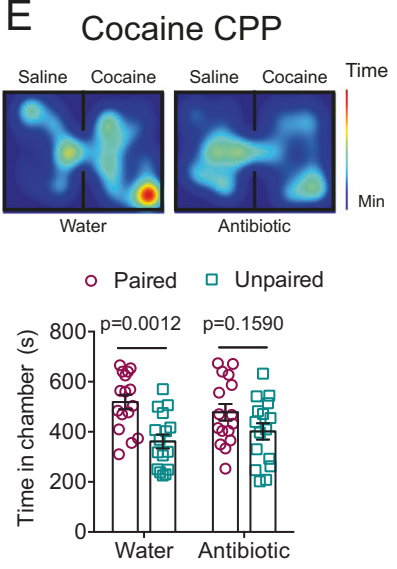

C

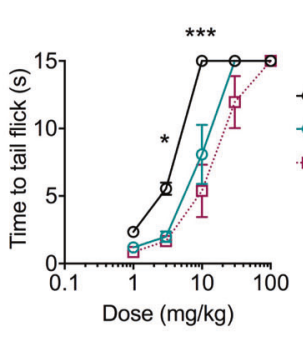

Tolerance

$\mathrm{F}$
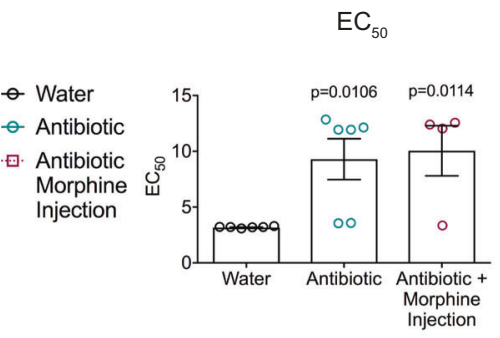

Hyperalgesia

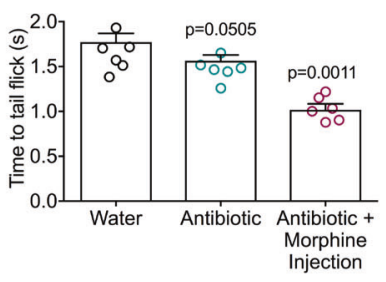

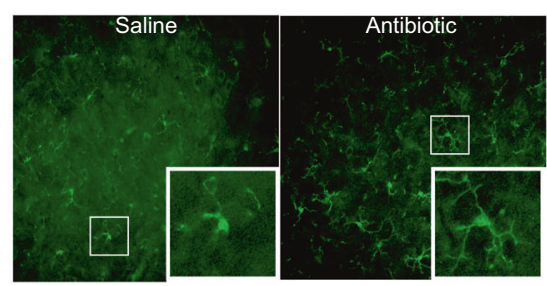

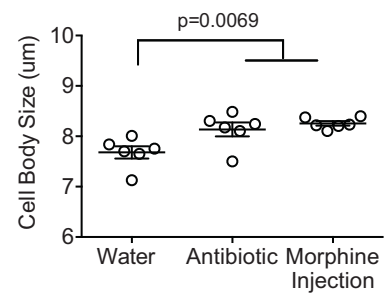

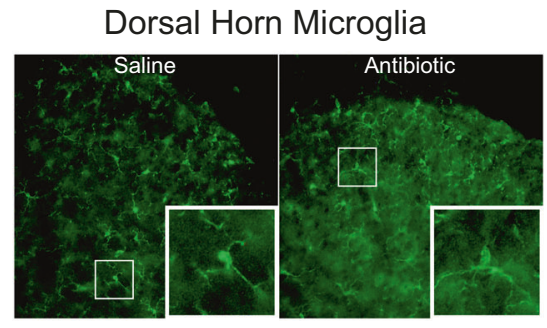

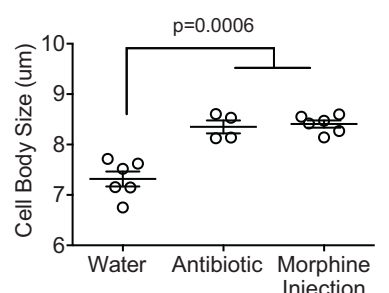

Fig. 3 Oral antibiotics alters microglial morphology and leads to hyperalgesia and impairments in reward behavior. a Timeline outlining experiments, starting with four days of antibiotic and opioid treatment followed by (1) evoked pain testing then immunostaining or (2) ten days of cocaine condition place preference testing, with antibiotic treatment continuing through both experiment. b Fecal microbiome diversity before and after antibiotic treatment c Impact of antibiotic treatment on morphine analgesia. Left: morphine dose-response curves of water-treated (control), antibiotic-treated, and antibiotic-treated + morphine injection groups compared using two-way ANOVA (error bars $=$ S.E.M., ${ }^{*} p=0.0291 .{ }^{* *} p<0.0001, n=6$ ). Right: Calculated $\mathrm{EC}_{50}$ for each group, and compared with Kruskal-Wallis test followed by post hoc Dunn's multiple comparisons test (error bars = S.E.M., $n=4-6$ ). $\mathbf{d}$ Baseline tail flick responses compared with Kruskal-Wallis test followed by post hoc Dunn's multiple comparisons test (error bars=S.E.M., $n=6$ ). e Impact of antibiotic treatment on cocaine-conditioned place preference (CPP). Top: representative heat maps illustrating time spent in either saline-paired or cocaine-paired chamber. Bottom: Cocaine place preference scores for each group compared with a one-way ANOVA followed by a post hoc Sidak's multiple comparison test. $\mathbf{f}$ Impact of antibiotic treatment on microglial morphology in the ventral tegmental area (VTA) and dorsal horn of the spinal cord. Top: representative immunomicrographs from water and antibiotic-treated groups (inset box $=50 \mu \mathrm{m}$ by $50 \mu \mathrm{m}$ ). Bottom: Quantification from 3-6 sections per animal. Groups were compared with one-way ANOVA tests followed by post hoc Dunn's multiple comparisons tests (error bars $=$ S.E.M., $n=6$ )

that microglial activation is a critical opponent process leading to hyperalgesia and deficits in dopamine-dependent reward responding $[5,9]$. While morphine and other opioids are reported to directly activate glia [29, 30], opioid use also disrupts the gut microbiota $[20,21]$. Thus, opioids may engage opponent processes via the gut-brain signaling axis that further exacerbate the pro-inflammatory effects of opioids [31]. In this study, we found that opioid-induced perturbations in the gut microbiota causally relate to neuroinflammation, deficits in reward responding, and hyperalgesia (Fig. 4). Importantly, we also found the repeated withdrawal periods of intermittent morphine exacerbated these anhedonic and hyperalgesic effects, possibly due to changes in gut microbiota.

Morphine was administered either intermittently with twicedaily morphine injections or continuously with a subcutaneous morphine pellet. Intermittent morphine injections were interspaced by periods of repeated withdrawal, while morphine pellet administration did not include any periods of withdrawal, allowing us to separate the effects of morphine withdrawal from the drug itself. Mice treated with either morphine injections or pellet displayed similar levels of tolerance. Microglial activation and impaired cocaine reward behavior were only observed in mice with intermittent morphine administration, or following morphine pellet removal. While opioids have been shown to have a direct effect on microglial activation [29], the current results suggest periods of withdrawal can exacerbate this central inflammatory response (Fig. 1). Given that conditions of chronic stress have been shown to significantly increase microglial activation, it suggests that repeated withdrawal may have acted as a chronic stressor [32]. Certainly, withdrawal from chronic opioids has been shown to elevate hypothalamic-pituitary-adrenal axis signaling, lending support to this hypothesis [33-36]. Additional studies are warranted to explore whether these different morphine treatment regimens impact other behaviors such as locomotion, anxiety, and depression.

The differences in phenotypic response between intermittent and sustained morphine regimens raises several important questions regarding the length of time and number of withdrawal periods needed to exacerbate the opioid-dependent phenotypes measured here. The subcutaneous morphine pellet used here is limited by the ability to manipulate drug plasma levels once implanted, and thus we are unable to address these questions at this time.

A complementary hypothesis raised in this study is that differences in intermittent and sustained morphine treatment may be related to changes in the gut microbiota. Alterations in the 

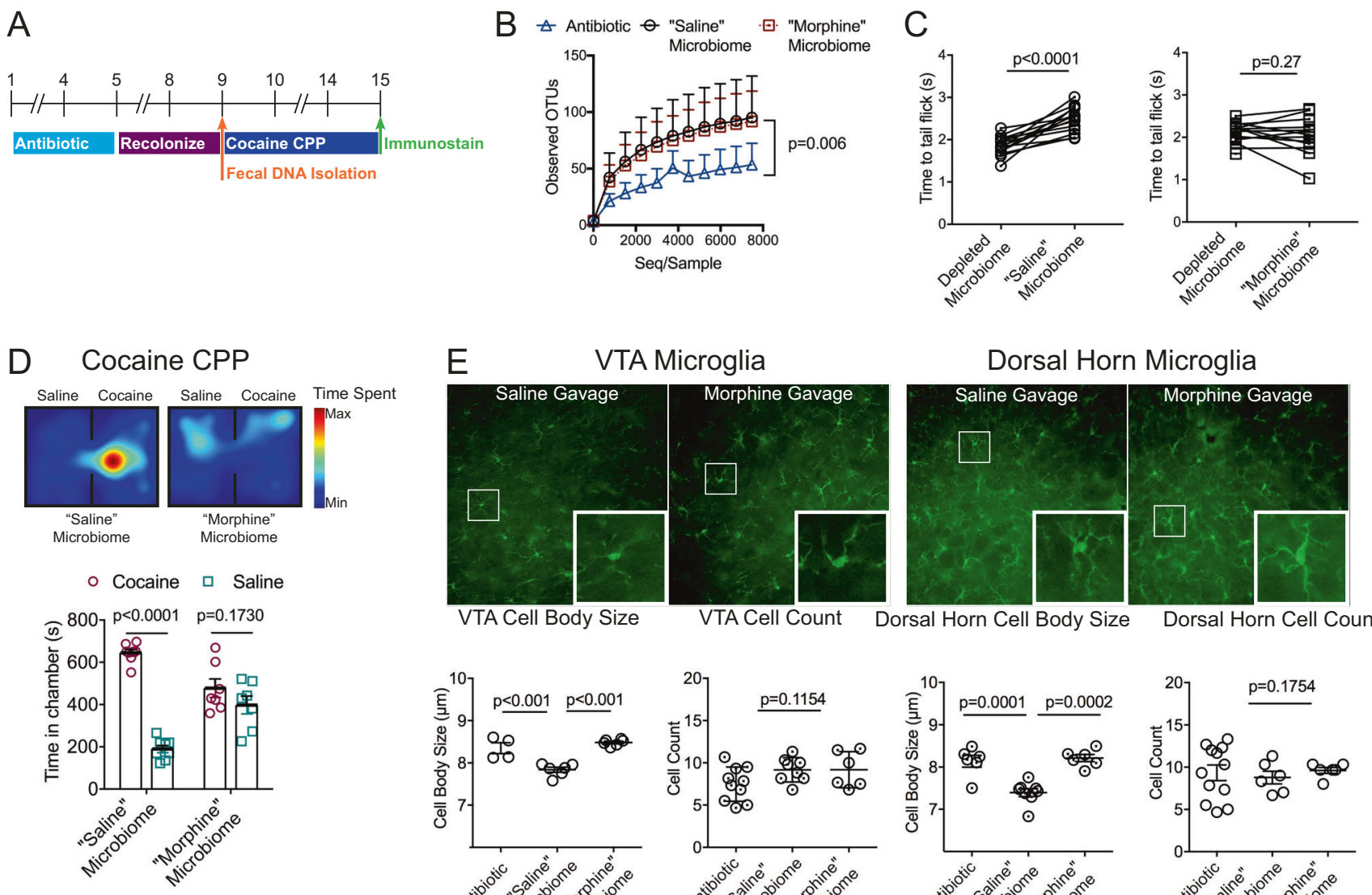

VTA Cell Body Size
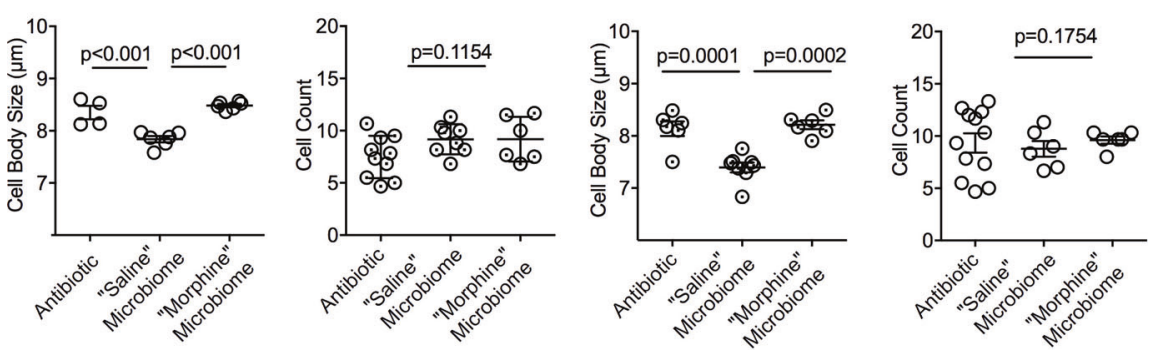

Fig. 4 Gut microbiome recolonization from naive mice donors, but not morphine-dependent donors, restores normal reward behavior, and microglia morphology in antibiotic-treated mice. a Timeline outlining experiments, starting with four days of antibiotic treatment, followed by four days of microbiome recolonization after oral gavage, fecal pellet collection, six days of cocaine-conditioned place preference, and immunostaining. b Fecal microbiome diversity following microbiome recolonization. c Baseline tail withdrawal after antibiotic treatment (microbiome depleted) and after oral recolonization with fecal pellets isolated from saline and intermittent morphine-treated mice. Groups compared with Student's t-test (error bars =S.E.M, $n=14$ ). d Cocaine place preference in saline and morphine recolonized groups. Top: representative heat maps illustrating time spent in either saline-paired or cocaine-paired chamber. Bottom: Quantification of cocaine place preferences. Groups compared with one-way ANOVA followed by a post hoc Sidak's multiple comparison test (error bars $=$ S.E.M, $n=6-8$ ). e Impact of microbiome recolonization on microglial morphology in the ventral tegmental area (VTA) and dorsal horn of the spinal cord. Top: representative immunomicrographs from animals orally gavaged with fecal pellets isolated from saline-treated or morphine-dependent host animals (inset box $=50 \mu \mathrm{m}$ by $50 \mu \mathrm{m}$ ). Bottom: Quantification of microglial morphology and number from 3-6 slices per animal. Groups were compared with Krukal-Wallis tests (error bars = S.E.M., $n=6-8$ )

gut microbiome are associated with anhedonic states, such as depression [16]. Moreover, bacteria indigenous to the gut directly contribute to the maturation and activation of microglia, and alterations in the gut microbiome are associated with neuroinflammation [13, 14]. Given that opioids significantly impact gut motility (either by decreasing gut motility during drug onset or increasing gut motility during withdrawal), we sought to assess how the different morphine treatment regimens (intermittent versus sustained) impacted the gut microbiota. We found that intermittent and sustained morphine treatment altered the taxonomic composition of gut bacteria. These changes were not only different from saline controls, but also between the different morphine regimens (Fig. 2). While intermittent morphine treatment significantly decreased the relative abundances of Lactobacillus spp. and increased Ruminococcus spp., these taxa were unaffected following sustained morphine treatment. In contrast, sustained morphine treatment showed significant increases in the genus Clostridium and family Rikenellaceae, and these differences that were not observed in the intermittent morphine-treated group (Fig. 2f). Chronic treatment with morphine slows gut motility leading to constipation, while withdrawal from opioids leads to increased gut motility and diarrhea $[17,18]$. Changes in gut motility can influence the gut microbiome; therefore, it is possible that chronic treatment with morphine may alter gut motility and microbiota as seen here.

The mechanism by which the disrupted gut microbiota influence central inflammation and behavior is not completely understood. Possible mechanisms include vagus nerve signaling, gut hormone signaling, and changes to the gut permeability [37]. Multiple studies have shown that gut microbes can influence affective behaviors through primary afferent pathways in the vagus nerve [38, 39]. Alternatively, the gut microbiota may influence inflammation and behavior through its influence on the integrity of the intestinal barrier $[40,41]$. Increased gut permeability can increase the translocation of bacterial components and their metabolic products across the intestinal wall [41]. This bacterial translocation can provoke local inflammatory cytokine release and vagus nerve activation, both of which can trigger an immune response within the peripheral and central nervous systems. This is supported by recent research that found chronic opioids increased intestinal permeability and bacterial translocation to peripheral tissues $[42,43]$, and by our current 
research that found intermittent morphine, but not sustained morphine, increased intestinal permeability (Fig. S1).

To assess the relationship between opioid-dependent phenotypes and gut dysbiosis, we depleted the gut microbiota of opioidnaive mice with oral antibiotics and measured changes in inflammation and opioid-related behavior. Depletion of the host microbiome via oral antibiotics unexpectedly resulted in altered microglia morphology, produced opioid tolerance, and impaired reward behaviors measured by cocaine CPP (Fig. 3), suggesting that antibiotic treatment can confer phenotypes associated with opioid dependence. The cocktail of antibiotics used in this study exhibit poor oral bioavailability [44]; only ampicillin shows moderate absorption from the gut, but does not cross the blood brain barrier [45]. Thus, effects of antibiotic treatment on central inflammatory cells are likely to be via direct manipulation of gut bacterial communities or a downstream consequence of such manipulation. Of note, previous literature suggests this interaction between microbiota depletion and opioid-dependent phenotypes is complex. For example, the effect of antibiotic treatment on opioid tolerance may depend on additional variables including length and type of treatment, as 10 day antibiotic treatment did not impact development of opioid tolerance [46]. Moreover, the effect of antibiotics on impaired reward behavior may also depend on additional variables, given that a previous study found antibiotic depletion of the gut microbiota either did not effect or enhanced cocaine CPP depending on the dose of cocaine used [47]. The above cited studies varied in the study species, dose and type of antibiotics, as well as length of treatment, all which may contribute to the variability in these results. Further research is necessary to parse out these variables, but all point towards a strong relationship between gut microbiota composition and reward-related phenotypes. Previous studies have shown that germ-free mice exhibit abnormal microglia morphology and immune response [13], and antibiotic treatment increases hippocampal BDNF in specific pathogen-free mice [48]. We have shown previously that chronic opioids elevate BDNF levels in the mesolimbic midbrain via activated microglia [9], and these studies suggest this may be driven, in part, by changes to the gut microbiota.

To assess the causal link between the gut microbiota and opioid-related phenotypes, we recolonized antibiotic-treated drug-naive mice with saline- or morphine-associated microbiota (Fig. 4). Drug-naive mice were initially depleted of their host microbiome by oral antibiotic treatment described above (Fig. 3). Following depletion, animals were recolonized with fecal samples from either saline control or opioid-dependent animals. Antibiotic treatment alone resulted in increased microglial cell body size and impaired reward behavior. However, antibiotic-treated animals transplanted with saline microbiota exhibited restored normal reward behavior and a resting microglial state (Fig. 4). Animals transplanted with morphine-associated microbiota had impaired reward behavior and activated microglia associated with antibiotic treatment or intermittent morphine treatment alone. This suggests that the altered gut microbiota following antibiotic or intermittent morphine is similarly detrimental to normal reward behavior and resting inflammatory state.

These results indicate that the regimen of morphine administration (intermittent versus sustained) is an important factor when discussing the development of tolerance, hyperalgesia, and negative affect. It suggests that periodic withdrawal associated with intermittent morphine treatment exacerbates the inflammation-driven behavioral phenotypes associated with prolonged opioid use. Moreover, it implicates gut microbiota as being a putative intermediary step in driving these adaptations. Therapies that restore or protect healthy gut microbiota may be important for alleviating the side effects of prolonged opioid use. For example, oral methyl-naltrexone is an opioid antagonist currently used for the treatment of opioid-induced constipation
[49], and has been shown to alleviate some of the negative symptoms of chronic opioids including constipation and hyperalgesia [50]. The evidence presented herein suggest gut-restricted opioid antagonists (such as methyl-naltrexone) may positively affect clinical outcomes of opioid treatment by blocking opioidinduced changes to the gut flora while maintaining analgesic efficacy.

\section{FUNDING}

We would like to thank the NIDA Drug Supply for the cocaine and morphine sulfate powder, as well as the morphine and control subcutaneous pellets. This work was supported the National Institutes for Health (DA40016 to AMWT and DA005010 to CJE), The American Pain Society (Future Leaders in Pain Research to AMWT), the Shirley and Stefan Hatos Foundation (KL, CJE, AMWT), the Alfred P. Sloan Foundation Fellowship in Neuroscience (EYH), and UPLIFT: UCLA Postdoctoral Longitudinal Investment in Faculty Award (K12GM106996 to HEV)

\section{ADDITIONAL INFORMATION}

Supplementary Information accompanies this paper at (https://doi.org/10.1038/ s41386-018-0211-9).

Competing interests: The authors declare no competing interests.

Publisher's note: Springer Nature remains neutral with regard to jurisdictional claims in published maps and institutional affiliations.

\section{REFERENCES}

1. Cahill C, Taylor A. Neuroinflammation-a co-occurring phenomenon linking chronic pain and opioid dependence. Curr Opin Behav Sci. 2017;13:171-7.

2. Cahill CM, Walwyn W, Taylor AM, Pradhan AA, Evans CJ. Allostatic mechanisms of opioid tolerance beyond desensitization and downregulation. Trends Pharmacological Sci. 2016;37:963-76.

3. Raghavendra V, Rutkowski MD, DeLeo JA. The role of spinal neuroimmune activation in morphine tolerance/hyperalgesia in neuropathic and shamoperated rats. J Neurosci. 2002;22:9980-9.

4. Raghavendra V, Tanga FY, DeLeo JA. Attenuation of morphine tolerance, withdrawal-induced hyperalgesia, and associated spinal inflammatory immune responses by propentofylline in rats. Neuropsychopharmacology. official publication of the American College of Neuropsychopharmacology 2004;29:327-34.

5. Ferrini F, Trang T, Mattioli TA, Laffray S, Del'Guidice T, Lorenzo LE, et al. Morphine hyperalgesia gated through microglia-mediated disruption of neuronal $\mathrm{Cl}(-)$ homeostasis. Nat Neurosci. 2013;16:183-92.

6. Burma NE, Bonin RP, Leduc-Pessah H, Baimel C, Cairncross ZF, Mousseau M, et al. Blocking microglial pannexin-1 channels alleviates morphine withdrawal in rodents. Nat Med. 2017;23:355-60.

7. Hutchinson MR, Lewis SS, Coats BD, Skyba DA, Crysdale NY, Berkelhammer DL, et al. Reduction of opioid withdrawal and potentiation of acute opioid analgesia by systemic AV411 (ibudilast). Brain Behav Immun. 2009;23:240-50.

8. Song $\mathrm{P}$, Zhao Z-Q. The involvement of glial cells in the development of morphine tolerance. Neurosci Res. 2001;39:281-6.

9. Taylor AM, Castonguay A, Ghogha A, Vayssiere P, Pradhan AA, Xue L, et al. Neuroimmune regulation of GABAergic neurons within the ventral tegmental area during withdrawal from chronic morphine. Neuropsychopharmacology. official publication of the American College of Neuropsychopharmacology 2016:41:949-59.

10. Romer Thomsen K, Whybrow PC, Kringelbach ML. Reconceptualizing anhedonia: novel perspectives on balancing the pleasure networks in the human brain Front Behav Neurosci. 2015;9:49.

11. Tye KM, Mirzabekov JJ, Warden MR, Ferenczi EA, Tsai HC, Finkelstein J, et al. Dopamine neurons modulate neural encoding and expression of depressionrelated behaviour. Nature. 2013;493:537-41.

12. Felger JC, Treadway MT. Inflammation effects on motivation and motor activity: role of dopamine. Neuropsychopharmacology: official publication of the American College of Neuropsychopharmacology. 2017;42:216-41.

13. Erny D, Hrabe de Angelis AL, Jaitin D, Wieghofer P, Staszewski O, David E, et al. Host microbiota constantly control maturation and function of microglia in the CNS. Nat Neurosci. 2015;18:965-77.

14. Sampson TR, Debelius JW, Thron T, Janssen S, Shastri GG, Ilhan ZE, et al. Gut Microbiota Regulate Motor Deficits and Neuroinflammation in a Model of Parkinson's Disease. Cell. 2016;167:1469-80.e1412. 
15. Cowan CSM, Hoban AE, Ventura-Silva AP, Dinan TG, Clarke G, Cryan JF. Gutsy moves: the amygdala as a critical node in microbiota to brain signaling. Bioessays. 2018;40:1700172.

16. Zheng P, Zeng B, Zhou C, Liu M, Fang Z, Xu X, et al. Gut microbiome remodeling induces depressive-like behaviors through a pathway mediated by the host's metabolism. Mol Psychiatry. 2016;21:786-96.

17. De Schepper HU, Cremonini F, Park MI, Camilleri M. Opioids and the gut: pharmacology and current clinical experience. Neurogastroenterol Motil. 2004; 16:383-94.

18. Koob GF, Maldonado R, Stinus L. Neural substrates of opiate withdrawal. Trends Neurosci. 1992;15:186-91.

19. Rhee SH, Pothoulakis C, Mayer EA. Principles and clinical implications of the brain-gut-enteric microbiota axis. Nat Rev Gastroenterol Hepatol. 2009;6:306-14.

20. Acharya C, Betrapally NS, Gillevet PM, Sterling RK, Akbarali H, White MB, et al. Chronic opioid use is associated with altered gut microbiota and predicts readmissions in patients with cirrhosis. Aliment Pharmacol Ther. 2017;45:319-31.

21. Banerjee S, Sindberg G, Wang F, Meng J, Sharma U, Zhang L, et al. Opioidinduced gut microbial disruption and bile dysregulation leads to gut barrier compromise and sustained systemic inflammation. Mucosal Immunol. 2016;9: 1418-28.

22. Wang F, Meng J, Zhang L, Johnson T, Chen C, Roy S. Morphine induces changes in the gut microbiome and metabolome in a morphine dependence model. Sci Rep. 2018;8:3596.

23. Reikvam DH, Erofeev A, Sandvik A, Grcic V, Jahnsen FL, Gaustad P, et al. Depletion of murine intestinal microbiota: effects on gut mucosa and epithelial gene expression. PLoS ONE. 2011;6:e17996.

24. Caporaso JG, Lauber CL, Walters WA, Berg-Lyons D, Lozupone CA, Turnbaugh PJ, et al. Global patterns of $16 \mathrm{~S}$ rRNA diversity at a depth of millions of sequences per sample. Proc Natl Acad Sci USA. 2011;108:4516-22.

25. Karperien A, Ahammer $H$, Jelinek HF. Quantitating the subtleties of microglial morphology with fractal analysis. Front Cell Neurosci. 2013;7:3.

26. Evans CJ, Cahill CM. Neurobiology fo opioid dependence in creating addiction vulnerability. F1000 Res. 2016;5:1-11.

27. Leri F, Zhou Y, Goddard B, Cummins E, Kreek MJ. Effects of high-dose methadone maintenance on cocaine place conditioning, cocaine self-administration, and muopioid receptor mRNA expression in the rat brain. Neuropsychopharmacology. official publication of the American College of Neuropsychopharmacology 2006;31:1462-74.

28. Harned M, Sloan P. Safety concerns with long-term opioid use. Expert Opin Drug Saf. 2016;15:955-62.

29. Hutchinson MR, Northcutt AL, Hiranita T, Wang X, Lewis SS, Thomas J, et al. Opioid activation of toll-like receptor 4 contributes to drug reinforcement. J Neurosci. 2012;32:11187-200.

30. Hutchinson MR, Zhang Y, Shridhar M, Evans JH, Buchanan MM, Zhao TX, et al. Evidence that opioids may have toll-like receptor 4 and MD-2 effects. Brain Behav Immun. 2010;24:83-95.

31. Mayer EA, Tillisch K, Gupta A. Gut/brain axis and the microbiota. J Clin Invest. 2015;125:926-38.

32. Tynan RJ, Naicker S, Hinwood M, Nalivaiko E, Buller KM, Pow DV, et al. Chronic stress alters the density and morphology of microglia in a subset of stressresponsive brain regions. Brain Behav Immun. 2010;24:1058-68.

33. Martinez-Laorden E, Hurle MA, Milanes MV, Laorden ML, Almela P. Morphine withdrawal activates hypothalamic-pituitary-adrenal axis and heat shock protein 27 in the left ventricle: the role of extracellular signal-regulated kinase. J Pharmacol Exp Ther. 2012;342:665-75.

34. Sudo N, Chida Y, Aiba Y, Sonoda J, Oyama N, Yu XN, et al. Postnatal microbial colonization programs the hypothalamic-pituitary-adrenal system for stress response in mice. J Physiol. 2004;558:263-75.

35. Buffington SA, Di Prisco GV, Auchtung TA, Ajami NJ, Petrosino JF, Costa-Mattioli M. Microbial reconstitution reverses maternal diet-induced social and synaptic deficits in offspring. Cell. 2016;165:1762-75.

36. Zhou Y, Leri F, Ho A, Kreek MJ. Suppression of hypothalamic-pituitary-adrenal axis by acute heroin challenge in rats during acute and chronic withdrawal from chronic heroin administration. Neurochem Res. 2013;38:1850-60.

37. Foster JA, Rinaman L, Cryan JF. Stress \& the gut-brain axis: regulation by the microbiome. Neurobiol Stress. 2017;7:124-36.

38. Goehler LE, Park SM, Opitz N, Lyte M, Gaykema RP. Campylobacter jejuni infection increases anxiety-like behavior in the holeboard: possible anatomical substrates for viscerosensory modulation of exploratory behavior. Brain Behav Immun. 2008;22:354-66.

39. Lyte M, Li W, Opitz N, Gaykema RP, Goehler LE. Induction of anxiety-like behavior in mice during the initial stages of infection with the agent of murine colonic hyperplasia Citrobacter rodentium. Physiol Behav. 2006;89:350-7.

40. Kelly JR, Kennedy PJ, Cryan JF, Dinan TG, Clarke G, Hyland NP. Breaking down the barriers: the gut microbiome, intestinal permeability and stress-related psychiatric disorders. Front Cell Neurosci. 2015;9:392.

41. Yarandi SS, Peterson DA, Treisman GJ, Moran TH, Pasricha PJ. Modulatory effects of gut microbiota on the central nervous system: how gut could play a role in neuropsychiatric health and diseases. J Neurogastroenterol Motil. 2016;22: 201-12.

42. Meng J, Yu H, Ma J, Wang J, Banerjee S, Charboneau R, et al. Morphine induces bacterial translocation in mice by compromising intestinal barrier function in a TLR-dependent manner. PLoS ONE. 2013;8:e54040.

43. Bhave S, Gade A, Kang M, Hauser KF, Dewey WL, Akbarali HI. Connexin-purinergic signaling in enteric glia mediates the prolonged effect of morphine on constipation. FASEB J. 2017;31:2649-60.

44. Matzke GR, Zhanel GG, Guay DR. Clinical pharmacokinetics of vancomycin. Clin Pharmacokinet. 1986;11:257-82.

45. Frohlich EE, Farzi A, Mayerhofer R, Reichmann F, Jacan A, Wagner B, et al. Cognitive impairment by antibiotic-induced gut dysbiosis: analysis of gut microbiotabrain communication. Brain Behav Immun. 2016;56:140-55.

46. Kang M, Mischel RA, Bhave S, Komla E, Cho A, Huang C, et al. The effect of gut microbiome on tolerance to morphine mediated antinociception in mice. Sci Rep. 2017;7:42658.

47. Kiraly DD, Walker DM, Calipari ES, Labonte B, Issler O, Pena CJ, et al. Alterations of the host microbiome affect behavioral responses to cocaine. Sci Rep. 2016;6: 35455.

48. Bercik P, Denou E, Collins J, Jackson W, Lu J, Jury J, et al. The intestinal microbiota affect central levels of brain-derived neurotropic factor and behavior in mice. Gastroenterology. 2011;141:599-609.

49. Nee J, Zakari M, Sugarman MA, Whelan J, Hirsch W, Sultan S, et al. Efficacy of treatments for opioid-induced constipation: a systematic review and metaanalysis. Clin Gastroenterol Hepatol. 2018;S1542-3565:30087-9.

50. Corder G, Tawfik VL, Wang D, Sypek El, Low SA, Dickinson JR, et al. Loss of mu opioid receptor signaling in nociceptors, but not microglia, abrogates morphine tolerance without disrupting analgesia. Nat Med. 2017;23:164-73. 